\title{
LA GESTIÓN DEL TALENTO PARA PRODUCIR Y GESTIONAR EL CAPITAL INTELECTUAL O CONOCIMIENTO EXPLÍCITO EN NUESTRAS ESCUELAS
}

Betty Alfaro Palacios*
Universidad Católica Sedes Sapientiae
ealfaro@ucss.edu.pe

Fecha de recepción: agosto de 2015 Fecha de aceptación: octubre de 2015

Resumen: El tema de este trabajo se enmarca dentro de la gestión del conocimiento y del capital intelectual. A partir de este último, profundizará

Betty Alfaro Palacios es magíster en Gestión de la Educación por la Pontificia Universidad Católica del Perú. Se desempeńa como docente en el pregrado y es jefa de la Unidad Central de Calidad Académica y Acreditación de la Universidad Católica Sedes Sapientiae. Ha ganado dos veces el Concurso Nacional "Horacio", promovido por la Derrama Magisterial en el área de investigación educativa, y el Concurso Programa de Apoyo a la Investigación para Estudiantes de Postgrados-PAIP-2006 de la Pontificia Universidad Católica del Perú. De igual modo, es coautora de las publicaciones Gestión del conocimiento. Una exigencia de la educación actual (Lima, Instituto de Formación y Desarrollo Docente de la Derrama Magisterial, 2009) y La formación en gestión de la educación. Tendencias y experiencias desde los postgrados (Lima, Pontificia Universidad Católica del Perú, 2008). 
en la gestión del conocimiento explícito para producir y compartir conocimiento organizacional que ayude a la escuela a ser una institución que aprende. Con ese objetivo, se expondrá un breve marco teórico sobre cada uno de los componentes utilizados en la investigación como son la gestión del conocimiento, el capital intelectual y la gestión del talento. El objetivo del trabajo fue exponer cómo los directivos tienen que saber gestionar los intangibles o conocimientos tácitos que producen los docentes y hacer que estos se conviertan en conocimiento tangible o explícito para que se pueda compartir. Para ello, se realizó una investigación en la Institución Educativa (IE) Fe y Alegría N. 2 del distrito de San Martín de Porres, Lima, Perú.

La investigación se realizó bajo el enfoque cualitativo, pues utilizó las técnicas de la observación, la entrevista y el análisis documental. Finalmente, los resultados ayudarán a entender la manera en que la institución estudiada puede ser un gran espacio donde llevar a cabo la conversión social del conocimiento. Asimismo, permitirá conocer de qué manera se logra el proceso de creación del conocimiento organizacional o capital intelectual en esta institución.

Palabras Clave: Gestión del conocimiento, capital intelectual, conocimiento tácito, conocimiento explícito, gestión de los intangibles o gestión del talento, creación del conocimiento, compartir conocimiento. 


\title{
THE MANAGEMENT OF THE TALENT TO PRODUCE AND TO MANAGE THE INTELLECTUAL CAPITAL OR EXPLICIT KNOWLEDGE IN OUR SCHOOLS
}

\begin{abstract}
Aвstract: The topic of this work places inside the management of the knowledge and of the intellectual capital. From the latter, it will penetrate into the management of the explicit knowledge to produce and to share knowledge organizational that helps to the school to be an institution that he learns. With this aim, a brief theoretical frame will be exposed on each of the components used in the investigation since they are the management of the knowledge, the intellectual capital and the management of the talent. The aim of the work was to expose how the executives have to be able to manage the intangibles or tacit knowledge that the teachers produce and do that these turn into tangible or explicit knowledge in order that it could share. For it, an investigation realized in the Educational Institution (IE) Fe y Alegría N. 2 of the district of San Martín de Porres, Lima, Peru.
\end{abstract}

The investigation was realized under the qualitative approach, since it used the technologies of the observation, the interview and the documentary analysis. Finally, the results will help to understand the way in which the studied institution can be a great space where to carry out the social conversion of the knowledge. Likewise, it will allow to know about what way achieves the process of creation of the knowledge organizational or the intellectual capital in this institution.

KEYwords: Management of the knowledge, the intellectual capital, tacit knowledge, explicit knowledge, management of the intangibles or management of the talent, creation of the knowledge, to share knowledge. 


\section{BETTY ALFARO PALACIOS}

\section{Introducción}

T a incorporación de categorías utilizadas en la teoría del management se muestra más que antes en el área de la educación. Términos como capital intelectual, gestión del capital intelectual, conocimiento organizacional, modos de conversión del conocimiento, constituyen un espectro teórico que puede ser útil para la mejora integral de un centro educativo. Por esa razón, en aras de una mejor comprensión del marco teórico utilizado, se explicará brevemente estas categorías utilizadas.

De igual forma, la aplicación de esta terminología puede ser útil de acuerdo al contexto limeño. Por ese motivo, el presente trabajo ha tomado como objeto de estudio la Institución Educativa Fe y Alegría N. 2, ubicado en el distrito limeño de San Martín de Porres. Este análisis, a su vez, fue de enfoque cualitativo, pues utilizó técnicas de la observación, la entrevista y el análisis documental. Finalmente, el uso en conjunto del marco teórico y metodológico utilizado, permitirá conocer de qué manera se logra el proceso de creación del conocimiento organizacional o capital intelectual en esta institución.

\section{Marco Teórico}

En las últimas décadas se han originado cambios en los entornos económicos, que han producido conceptos como globalización, innovación, nueva economía, gestión del conocimiento (KM), gestión del capital intelectual (ICM), capital humano y capital estructural, entre otros. Estos términos vienen siendo incorporados a las teorías del management y de la economía. Con ello, se evidencia la búsqueda de soluciones para la gestión de las organizaciones orientadas a la creación de valor (Pricewaterhousecoopers, 2000). 


\section{LA GESTIÓN DEL TALENTO PARA PRODUCIR Y GESTIONAR EL CAPITAL INTELECTUAL O CONOCIMIENTO EXPLÍCITO EN NUESTRAS ESCUELAS}

Dos de estos conceptos, la gestión del conocimiento y la gestión del capital intelectual, se confunden, y sus contenidos, en parte, se superponen. Sin embargo, existen diferencias de enfoque entre ellos. Es oportuno, por tanto, tener claros ambos conceptos.

\subsection{Gestión del conocimiento}

Gran parte de la literatura revisada confirma lo que se afirmó en el párrafo anterior y, por ello, la definición presentada por Vásquez (2005) ayudará a comprender este concepto. Según este autor, "la gestión del conocimiento es el esfuerzo de una organización por conseguir, capturar, organizar, compartir y distribuir los conocimientos entre los empleados" (p. 31). Por otra parte, es pertinente considerar las actividades planteadas por Vásquez en el proceso de gestión del conocimiento, debido a que son las que van a permitir crear conocimiento organizacional, e identificar el conocimiento explícito o tangible. Estas actividades son las que siguen:

- Capturar conocimiento, que se refiere a documentar las mejores prácticas de la organización, la resolución de problemas técnicos y los proyectos, entre otros.

- Almacenar conocimiento en archivos, de tal manera que pueda encontrarse fácilmente.

- Compartir, distribuir y dar acceso al conocimiento, con el fin de hacerlo llegar a quien lo necesita, o que las personas accedan fácilmente a él.

- Utilizar el conocimiento, lo que recuerda que, por lo menos en la organización, el conocimiento debe servir para la acción, es decir, para resolver problemas y tomar decisiones. 


\section{BETTY ALFARO PALACIOS}

Numerosos expertos que trabajan en sus organizaciones con el recurso conocimiento, como Kelleher (2000), se preguntan "why Knowledge Management (KM)?”. La respuesta para él está en que las organizaciones, en el lugar donde se encuentren, tienen que responder a enormes cambios que requieren de la gestión del conocimiento (KM) como una estrategia basada en los siguientes aspectos: (a) la cultura, (b) los contenidos, (c) la infraestructura, (d) el apoyo, (e) la visión y (f) el pensamiento de los líderes. Pricewaterhousecooper (2000) coincidió con Kelleher cuando afirmó que la gestión del conocimiento requiere de un cambio de cultura en las personas, en las organizaciones y en las estructuras del sistema económico. Esto exige a las organizaciones — especialmente a la educativa — innovación, aprendizaje y adaptación.

Por otro lado, en sintonía con Hargreaves (2003), se afirma que los docentes tienen que comprender que el mundo en el que enseñan está experimentando profundas transformaciones y que si se resignan a que la educación pública sea de bajo coste contribuyen a que la labor docente se limite a mantener el orden y enseñar para los exámenes. De esta manera, durante las próximas décadas, los docentes no serán capaces de enseñar para la sociedad del conocimiento, como se plantea en el actual DCN (Diseño Curricular Nacional [DCN], 2005) en uno de los objetivos de la Educación Básica Regular (EBR).

Según afirmó Capella (2005), el conocimiento como principal objeto de trabajo del administrador y del educador presenta aún serios vacíos. Sin embargo, en la escuela se debe aprender a gestionar el conocimiento, porque, actualmente, la sociedad es diferente, como afirmó Hargreaves (2003). En esta nueva sociedad hay altos riesgos debido a la violencia y a la devastación medioambiental a gran escala. Por lo tanto, el conocimiento en la educación para esta sociedad no solo tiene que ver con el aprendizaje 


\section{LA GESTIÓN DEL TALENTO PARA PRODUCIR Y GESTIONAR EL CAPITAL INTELECTUAL O CONOCIMIENTO EXPLÍCITO EN NUESTRAS ESCUELAS}

cognitivo, sino también con el desarrollo de una serie de capacidades contempladas en el DCN de EBR, tales como la formación de la ciudadanía y de la identidad, la equidad de género, entre otras.

\subsection{Gestión del capital intelectual}

La gestión del capital intelectual, según Sullivan (2001), es el proceso mediante el cual las empresas crean valor a partir de su capacidad intelectual o activos intelectuales. Estos son recursos intangibles capaces de crear valor, y necesarios para conseguir los objetivos de la empresa. En este sentido, la gestión del capital intelectual tiene un fin estratégico. Se debe considerar, como afirmó TFPL Ltd. (1999), que "Knowledge economies are emerging in the western world where knowledge, expertise, and innovation are now primary asset and key competitive advantage. To be successful in a knowledge economy organizations need to change and adapt" (p. 9).

Asimismo, los docentes, como trabajadores de este tipo de organización, tienen que acceder y comprender a la sociedad del conocimiento en la cual viven y trabajan los estudiantes. En caso contrario, se corre el riesgo de no prepararlos para que se desenvuelvan en ella. Prepararse como docentes para desenvolverse en esta sociedad, implica ser creativos e innovadores, lo cual exige tener al conocimiento como recurso generador de innovaciones pedagógicas y de gestión. Estas innovaciones promoverán una educación de calidad como se exige desde la Ley General de Educación 28044 (2003).

Todo esto conduce al planteamiento de una pregunta fundamental: ¿Cómo deben gestionar los directivos para que las escuelas capturen, almacenen, compartan y utilicen conocimiento? Según Vicario (2002), un sistema de administración de conocimientos en el ámbito educativo 


\section{BETTY ALFARO PALACIOS}

nos permitiría una buena administración de los bancos de información y contenidos. Además, se capitalizaría el conocimiento como producto de los procesos académicos, ofreciendo la posibilidad de aumentar el talento de los integrantes de la comunidad educativa.

En resumen, en la gestión del capital intelectual (ICM) el objetivo principal es crear valor para alcanzar los objetivos de la empresa (Sullivan, 2001). En el caso educativo, se debe gestionar el capital intelectual de la escuela para lograr el objetivo fundamental de la educación, establecido en la Ley General de Educación 28044:

Formar personas capaces de lograr su realización ética, intelectual, artística, cultural, afectiva, física, espiritual (...) para el ejercicio de su ciudadanía en armonía con su entorno... para vincular su vida con el mundo del trabajo y para afrontar los incesantes cambios en la sociedad y el conocimiento.

Por último, es importante tener en cuenta que la gestión del conocimiento (KM) y la gestión del capital intelectual (ICM), se integran y se requieren mutuamente. Así lo afirmó Wiig:

From a broader perspective, ICM and KM are fundamental building blocks -even cornerstones- in the effective management model for the 21 st century (...) ICM and KM cannot be pursued in isolation. They must be interwoven with other management considerations to make a sound, balanced and competitive enterprise. (Wiig, 1997, p. 400) 


\section{LA GESTIÓN DEL TALENTO PARA PRODUCIR Y GESTIONAR EL CAPITAL INTELECTUAL O CONOCIMIENTO EXPLÍCITO EN NUESTRAS ESCUELAS}

\subsection{Bases teóricas para la gestión del conocimiento en las escuelas}

Es importante tomar en cuenta lo que afirmó Gimeno (2001), quien manifestó que la educación es contemplada como un motor e instrumento importante para la realización del destino del ser humano y de la sociedad. Al respecto, el Proyecto Regional de Educación para América Latina y el Caribe (PRELAC, 2004) ha incorporado focos estratégicos para cumplir con las metas establecidas en Educación para Todos (EPT). Uno de los focos es el de la gestión y la flexibilización de los sistemas educativos para que la escuela pueda ofrecer oportunidades de aprendizaje efectivo a lo largo de toda la vida de las personas. Esto supone la transformación de la organización educativa a los tiempos que se viven actualmente.

Estas afirmaciones llevan a pensar, de desear que lo anteriormente expuesto se pueda cumplir, es indispensable partir de ciertos enfoques para entender los motivos de querer que la educación se oriente de una u otra forma (Gimeno, 2001). Es decir, debe partirse de paradigmas teleológicos que den sentido a las acciones y plantear para qué sirve. De esa forma, las preguntas a partir de este trabajo de investigación vendrían a ser ¿para qué sirve el capital intelectual en la organización educativa?, ¿con qué mirada está asumiéndose?

Es en esta dimensión teleológica que se asume el humanismo y el enfoque socio-crítico como las directrices que van construyendo el camino que indica por dónde debe transcurrirse en este cambio de época que nos ha tocado vivir; y encaminen nuestros propósitos hacia la mejora de la sociedad, de la cultura y de los sujetos. Vieujean (1959) afirmó que una de las normas que debe inspirar la educación, debe ser la de centrar la atención en las profundidades íntimas de la personalidad, considerando tanto el aspecto cognitivo como el espiritual. Es decir debe preocuparse de lo interno del ser 


\section{BETTY ALFARO PALACIOS}

humano y de la interiorización de la influencia educativa, y buscar lo que se afirma en el Informe de seguimiento de EPT en el mundo, 2005:

(...) la mejora de las competencias cognitivas, que es el objetivo universal y permanente de todo sistema educativo (...) en el hecho de que todas las sociedades consideran que la educación está llamada a fomentar comportamientos, actitudes y valores que se estiman necesarios para el civismo y la participación efectiva en la vida de la comunidad. (p. 2)

Por otro lado, el humanismo considera como otra función de la inteligencia al juicio moral (Claudel citado en Vieujean, 1959). Esta función debe fomentar los comportamientos, actitudes y valores que se plantean en el mismo informe y en el DCN de EBR (2005). A su vez, estos se deben ir construyendo desde el aula

Desde el planteamiento que hace Vieujean (1959), al afirmar que la cultura del sentido moral está descuidada en la educación moderna, se puede entender que esto es un imperativo que el humanismo viene planteando desde hace mucho tiempo y que en la actualidad se reafirma como uno de los "Focos estratégicos" del PRELAC (2004) y como uno de los aspectos esenciales que sustenta el DCN (2005). Es decir, deben darse nuevas miradas a viejos problemas. Asimismo, se tiene que hacer hincapié en los pilares de aprendizaje del Informe Delors, sobre todo en el de "aprender a ser", que constituye una excelente orientación para interrogarnos hacia dónde va la educación.

Es en estas afirmaciones que pueden encontrarse las redes posibles de formarse y movilizar tanto a la sociedad, en general, como a la organización educativa, en particular, para hacer de la educación una educación de calidad con equidad, tal como se afirma en la Ley General de Educación N. 28044. 


\section{LA GESTIÓN DEL TALENTO PARA PRODUCIR Y GESTIONAR EL CAPITAL INTELECTUAL O CONOCIMIENTO EXPLÍCITO EN NUESTRAS ESCUELAS}

De ese modo, generaría oportunidades de aprendizajes de calidad para todos y fomentaría una sociedad capaz de formar ciudadanos para una nación democrática. Dicha propuesta también fue planteada desde el Proyecto Educativo Nacional (PEN) en el 2005.

Estas dos miradas, humanismo y enfoque socio-crítico - - o individual y lo social - a criterio del presente estudio se complementan y pueden dar un sustento teórico a la Gestión del conocimiento en la escuela. La escuela, como una organización — que crea valor - en esta sociedad global, y del conocimiento en el nuevo entorno laboral, no puede escapar a los cambios vertiginosos del contexto. Asimismo, tiene que responder a su función principal: la formación integral del ser humano.

Desde esta mirada, es posible plantear la siguiente interrogante: ¿Cómo gestionar las instituciones educativas en este contexto para lograr los objetivos de la educación? A juicio de este trabajo, la Gestión de Recursos Humanos, desde el enfoque de la Gestión del Talento, puede ayudar a responder esta pregunta.

2.3.1 La Gestión de los recursos humanos: la gestión del talento para producir conocimiento tangible

Como se ha afirmado, los recursos de mayor importancia para la gestión de las organizaciones son los conocimientos y las capacidades. Estos solo lo poseen las personas, es decir los trabajadores de las organizaciones. El recurso humano se ha convertido en esta sociedad y economía del conocimiento en la pieza clave para innovar y alcanzar de esta manera la visión u objetivos de toda organización. Ahora bien, servirá en esta postura la siguiente afirmación de Grant: 


\section{BETTY ALFARO PALACIOS}

Recognition that the major source of knowledge is the expertise and know-how of employees has directed attention to human resource planning and appraisal. For example, "competency modelling" is used to identify the knowledge requirements of different occupations and guide appraisal and training. (1997, p. 451)

Esto indica que cada vez más las empresas dirigen su mirada hacia los recursos humanos que poseen y hacia el talento o competencias de estos para realizar la tarea. En otras palabras, toman en cuenta su competencia y su know-how, como afirma Grant. A su vez, el mismo autor cita como ejemplo el conocimiento y las competencias que se requieren para las diferentes ocupaciones en las organizaciones y cómo algunas empresas desde la Dow Chemical, Polaroid y Skandia, entre otras, están elaborando amplios sistemas de creación de conocimiento organizacional bajo la dirección de un director de "conocimiento" o "capital intelectual".

En efecto, la sociedad y la economía del conocimiento están promoviendo la inclusión en la organización de procesos que les ayuden a lograr sus objetivos. Uno de ellos es la gestión del talento (Jericó, 2001). Según esta autora "el talento es el recurso estratégico para innovar" (p. 7). Otro término usado en este proceso es la gestión por competencias Alles (2005), en la que se considera a la competencia como sinónimo de talento. Para la autora la competencia: "Hace referencia a las características de la personalidad, expresadas en comportamientos que generan un desempeño exitoso en el puesto de trabajo" (p. 29). Por ello, es necesario que cada persona integrante de la organización ocupe el lugar adecuado según su talento o competencias. 


\section{LA GESTIÓN DEL TALENTO PARA PRODUCIR Y GESTIONAR EL CAPITAL INTELECTUAL O CONOCIMIENTO EXPLÍCITO EN NUESTRAS ESCUELAS}

Según Jericó (2001), el talento requiere de una organización que facilite interacciones que den por resultado innovaciones. Entonces, es urgente, en primer lugar, que se identifiquen a los docentes con talento. En segundo término, tienen que facilitarse estas interacciones para que puedan surgir innovaciones.

Otra de las condiciones de la gestión del talento es el compromiso en el que está considerada la motivación que adoptan los trabajadores. Según Jericó (2001), este es el motor que hace que el trabajador, el docente en nuestro caso, aporte lo máximo de él hacia su institución educativa. Es decir, que se involucre "de lleno" con los objetivos de la educación para generar el capital intelectual que la escuela necesita.

A criterio de este trabajo, es importante resaltar la característica del talento propuesta por Jericó y es que según ella el talento se desarrolla. Desde este punto de vista, el talento es humanamente alcanzable y todos pueden lograrlo a través de un proceso de capacitación, que requiere de tiempo y ejercicio. Como afirmó Umbral: "El talento en buena medida, es una cuestión de insistencia” (citado en Jericó, 2001, p. 70).

Aquí juega un rol importante el desarrollo de las capacidades a través del ejercicio o de programas de formación. En consecuencia, a juicio de este estudio y en concordancia con Alles (2005), una de las tareas que debe emprender la organización en el área de recursos humanos es la de capacitación. Entendiendo, a su vez, por capacitación, el colaborar con los empleados para que estos hagan mejor su tarea.

A partir de esto, la formación de las personas dentro de las organizaciones cobra un valor estratégico. El aporte que puedan realizar quienes tengan a cargo la función de capacitación será ayudar a que los docentes, en el caso de las instituciones educativas, adquieran las capacidades necesarias para ayudarles a desempeñar mejor su tarea. Esto 


\section{BETTY ALFARO PALACIOS}

fomentaría la creación de capital intelectual con el que puedan lograr los objetivos estratégicos que se plantean en su Proyecto Educativo Institucional (PEI). Finalmente, puede decirse que la gestión del talento o gestión por competencias, requiere de un espacio que ayude a gestionar el capital intelectual producido en cualquiera de los puntos del proceso educativo.

\section{Creación e Identificación del Conocimiento o Capital Intelectual}

\subsection{El capital intelectual}

En este apartado se estudiarán los factores que aumentan el valor de una empresa u organización. En el caso de la organización educativa, dada su finalidad, este valor se traduce en la calidad educativa con equidad que se plantea desde la Ley General de Educación 28044. Se trata del “(...) nivel óptimo de formación que deben alcanzar las personas para enfrentar los retos del desarrollo humano, ejercer su ciudadanía y continuar aprendiendo durante toda la vida" (p. 9) y que las organizaciones educativas expresan en su Proyecto Educativo Institucional a partir de este planteamiento.

En el mundo súper competitivo de hoy — caracterizado por el progreso de las nuevas tecnologías de la información y la comunicación— los activos más valiosos de las empresas ya no son los activos tangibles tales como las maquinarias, edificios, instalaciones, y depósitos en los bancos. Al contrario, el valor recae ahora en los activos intangibles o capital intelectual (Sullivan, 2001). El conocimiento es ahora el creador de riqueza, el recurso estratégico más importante y el aprendizaje es el proceso vital de la nueva sociedad. Esto indica qué es lo que la institución debe fomentar para alcanzar la calidad educativa con equidad.

Por otro lado, es importante definir primero que cuando se habla de activos intangibles, se hace referencia a aquellos recursos que se basan en 


\section{LA GESTIÓN DEL TALENTO PARA PRODUCIR Y GESTIONAR EL CAPITAL INTELECTUAL O CONOCIMIENTO EXPLÍCITO EN NUESTRAS ESCUELAS}

capacidades y conocimientos. De igual forma, son capaces de producir valor para la organización. Estos se expresan en productos y servicios aunque no tienen existencia material, como afirmaron Edvinsson y Malone (1999), que son los más valiosos frente a los que se pueden adquirir con dinero (Solé \& Olea de Cárdenas, 2003).

\subsection{Concepto de capital intelectual}

Son muchas las definiciones que podemos encontrar sobre el capital intelectual en la literatura actual. En este caso, se partirá de la definición clásica del mismo. El capital intelectual según Sullivan (2001) "se puede entender simplemente como conocimiento que se puede convertir en beneficios" (p. 27). Es decir, el investigador se refiere a los beneficios de la creación de valor para la organización.

Se tomará en cuenta, asimismo, la definición de capital intelectual plasmada en la teoría. Esta fue establecida por Edvinsson y Malone (1999), quienes sostuvieron que se trata de cierta posesión que otorga una ventaja competitiva. Así, el "capital intelectual es la posesión de conocimientos, experiencia aplicada, tecnología organizacional, relaciones con clientes y destrezas profesionales (...) que dan ventaja competitiva en el mercado" (p. 64).

Desde la perspectiva del presente trabajo, esta definición es idónea para la escuela, dado que este tipo de organización, aunque con diferentes objetivos a la empresa, cuenta con recursos intangibles individuales y organizativos. Incluso, mantiene relaciones funcionales con entidades de la sociedad. Aquello con el fin de asegurar, como se plantea en la Ley General de Educación 28044, que el aprendizaje sea pertinente, integral y que potencie al servicio educativo. 


\section{BETTY ALFARO PALACIOS}

El capital intelectual forma empresas intensivas en conocimiento (Sveiby, 2000). En estas empresas la mayoría de los empleados son altamente cualificados y muy bien preparados, es decir, son profesionales del conocimiento. En este entendido, la escuela debería ser una de ellas, "como foco de innovación sustentado en el aprendizaje colaborativo", (Consejo Nacional de la Educación 2005, p. 47).

Por su función, el conocimiento es el recurso "propio" de estas organizaciones, y los docentes como profesionales que laboran en las escuelas, como empresas intensivas del conocimiento, deberían ser altamentecualificados. Esta exigencia se establece desde el Informe mundial de seguimiento de EPT en el mundo (2005). Por otro lado, el conocimiento entendido como la información ordenada, clasificada, sistematizada y "orientado hacia la acción" (Sveiby, 2000, p. 68), es el que ayuda a las empresas a innovar; por lo tanto, se requiere hacer efectivo el objetivo 3 del PEN: "Maestros bien preparados, que innoven y que ejerzan profesionalmente la docencia” (Consejo Nacional de Educación, 2005, p. 81).

\subsection{Modos de conversión del conocimiento}

La base de creación del conocimiento organizacional es la conversión del conocimiento tácito (el que está dentro de las personas) en conocimiento explícito y viceversa. El conocimiento explícito es el conocimiento que se puede expresar con palabras y números. Por ese motivo, se puede comunicar y compartir con facilidad en forma de "datos concretos, fórmulas científicas, procedimientos codificados o principios universales" (Nonaka \& Takeuchi citados en Wei, 1999, p. 144).

Por lo tanto, la creación del conocimiento de la organización es un proceso que amplía el conocimiento creado por los individuos. Según Nonaka 


\section{LA GESTIÓN DEL TALENTO PARA PRODUCIR Y GESTIONAR EL CAPITAL INTELECTUAL O CONOCIMIENTO EXPLÍCITO EN NUESTRAS ESCUELAS}

y Takeuchi (citados en Wei, 1999), existen dos dinámicas que impulsan el proceso de amplificación del conocimiento. En primer lugar, convertir el conocimiento tácito en explícito. En segundo lugar, llevar el conocimiento desde el nivel individual hasta los niveles de grupo, de organización e interorganizacional.

En esa línea, los modos de conversión del conocimiento son cuatro. Estos son los que siguen: (a) la socialización, (b) la exteriorización, (c) la combinación y (d) la interiorización. A continuación, veremos brevemente cada uno de ellos.

\subsubsection{La socialización: desde el conocimiento tácito hasta el conocimiento tácito}

La socialización es el proceso de compartir experiencias para ir creando conocimiento tácito, tal como modelos mentales y experiencias técnicas. Este modo comienza con la creación de espacios de interacción o reuniones entre los individuos lo que facilita el intercambio de experiencias. Estos espacios o reuniones también suelen ser informales. Mediante la socialización, un individuo puede adquirir conocimientos directamente de otros sin hacer uso del lenguaje, solo con base en la observación participante, al ayudar o imitar las conductas de profesionales experimentados, teniendo en cuenta que "the key to acquiring tacit knowledge is experience" (Nonaka \& Takeuchi, 1999, p. 63). Por tanto, la socialización transfiere conocimiento tácito a través de la experiencia que se comparte.

En la escuela, se podría aplicar este modo. Así, existirían dos momentos para que esto suceda. Estos son los siguientes: (a) primero, se ubica a docentes expertos y (b) segundo, se establecen rutinas de observación entre pares que le permitan a los docentes novatos aprender de sus compañeros expertos. 


\section{BETTY ALFARO PALACIOS}

3.3.2 La exteriorización: desde el conocimiento tácito hasta conocimiento explícito

Este es el proceso de codificar, sistematizar o convertir el conocimiento tácito en conceptos explícitos - escribir es un acto de convertir el conocimiento tácito en explícito- haciendo uso de metáforas, conceptos, analogías, hipótesis o modelos que orientan el logro de los

objetivos estratégicos de la organización. La exteriorización es la actividad fundamental en la creación del conocimiento organizacional a pesar de que como afirman los autores, esta ha sido descuidada. Mediante este proceso el conocimiento tácito se convierte en explícito a través de productos o servicios, es decir lo intangible se vuelve tangible, generando de esta manera capital intelectual a través de la innovación.

Este proceso se activa por el diálogo y la reflexión colectiva. En el campo de la educación siguiendo el ejemplo citado anteriormente el ejercicio de observación de las clases entre pares, daría lugar a reuniones para discutir y reflexionar sobre lo observado, y luego sistematizar la rutina. De esta manera se podría establecer una innovación y la escuela se podría convertir en una "escuela docente". Este ejercicio podría promover el uso de algunas metáforas como: "Desde el Ministerio de Educación hasta el aula" para el concepto de diversificación.

3.3.3 La combinación: desde el conocimiento explícito hasta el conocimiento explícito

Este es un proceso de combinar o reconfigurar el conocimiento explícito existente para producir nuevo conocimiento explícito expresado en servicios o productos. Este modo de conversión del conocimiento "involves 


\section{LA GESTIÓN DEL TALENTO PARA PRODUCIR Y GESTIONAR EL CAPITAL INTELECTUAL O CONOCIMIENTO EXPLÍCITO EN NUESTRAS ESCUELAS}

combining different bodies of explicit knowledge" (Nonaka \& Takeuchi, 1995, p.67). Los empleados de la organización intercambian y combinan conocimiento a través de conversaciones telefónicas, reuniones, memorandos o comunicaciones a través de internet, para encontrar contradicciones y reconfigurar la información.

A partir de ello, se puede categorizar, añadir, clasificar y confrontar cierta cantidad de información en una base de datos para producir nuevo conocimiento explícito. Aquí juegan un rol importante los directivos "medios" de la organización permitiendo que se produzca el intercambio del conocimiento a través de un trabajo en red. Debemos tener presente que tanto la exteriorización como la combinación son los procesos básicos para la creación del conocimiento explícito.

3.3.4 La interiorización: desde el conocimiento explícito hasta el conocimiento tácito

Este es el proceso de incorporación del conocimiento explícito en conocimiento tácito, interiorizando las experiencias obtenidas a través de los otros modos de la creación del conocimiento. La interiorización se facilita si el conocimiento queda capturado en documentos, manuales, historias orales, de modo que los individuos puedan volver a experimentar indirectamente la experiencia de otros. Según Nonaka y Takeuchi, la interiorización se relaciona con "learning by doing" (Nonaka \& Takeuchi, 1995, p.71).

Los documentos o manuales facilitan la transferencia de los conocimientos explícitos a otras personas. La actividad planteada en este modo se relaciona estrechamente con el capturar conocimiento o documentar las mejores prácticas que propuso Vásquez (2005) en la gestión del conocimiento. En este modo de creación del conocimiento, se pone en 
evidencia el uso del capital estructural en las organizaciones y en particular en las instituciones educativas, lo que da la posibilidad de crear nuevamente conocimiento tácito. Estos cuatro modos de creación del conocimiento interactúan y producen la espiral continua de creación del conocimiento de la organización.

\section{Marco Metodológico}

El enfoque de la investigación es cualitativo, dado que la intención de este trabajo es sobre todo describir y analizar para comprender (Cardona, 2002; Vieytes, 2004) cómo se crea el conocimiento organizacional. Asimismo, se busca responder a la siguiente pregunta: ¿cómo identificar el conocimiento tangible en la organización educativa Fe y Alegría N. 2? Todo ello se propone "desde la perspectiva de los mismos sujetos que lo producen y experimentan" (Vieytes, 2004, p. 613).

De igual forma, siguiendo la postura de Hernández (1998), el estudio se ubica dentro de la investigación no experimental. La razón es que se realizó sin manipular las categorías de investigación y se observó el fenómeno en su contexto natural para después analizarlo a la luz de la teoría. En cuanto al nivel o tipo de investigación, el estudio se encuadra dentro del nivel exploratorio (Hernández, 1998; Vieytes, 2004), ya que constituye un primer acercamiento al tema en estudio.

Dentro de lo que sostuvo Hernández: "Los estudios exploratorios sirven para familiarizarnos con fenómenos relativamente desconocidos, obtener información sobre la posibilidad de llevar a cabo una investigación más completa(...) establecer prioridades para investigaciones posteriores" (1998, p. 59). Esto resulta relevante porque que el presente estudio servirá para familiarizar al entorno educativo con un tema que está siendo 


\section{LA GESTIÓN DEL TALENTO PARA PRODUCIR Y GESTIONAR EL CAPITAL INTELECTUAL O CONOCIMIENTO EXPLÍCITO EN NUESTRAS ESCUELAS}

imprescindible en las escuelas — la gestión del Capital Intelectual- y "afinar problemas" (Vieytes, 2004, p. 612) con la posibilidad de realizar posteriores investigaciones "más rigurosas" en esta línea (Dankhe citado en Hernández, 1998, p. 59) que ayuden a crear valor, que en el caso de la educación se refiere a la mejora de la calidad educativa.

Los resultados presentados responden a la siguiente pregunta planteada: ¿cómo crear el conocimiento intelectual o conocimiento explícito en la I.E. Fe y Alegría N. 2 de San Martín de Porres? Asimismo, la población de la investigación estuvo formada por miembros de dicha institución. Estos son los que siguen: (a) tres directivos, que constituyen el "top" de la organización; (b) 22 maestros y maestras de inicial y primaria y 32 de secundaria y (c) ocho comités de gestión.

La elección de la muestra se hizo por muestreo aleatorio simple, porque "cada miembro de la población tiene la misma oportunidad de ser seleccionado para componer la muestra" (Cardona, 2002, p. 142), teniendo en cuenta que la población no es muy grande. En cuanto a la muestra tanto de individuos como de espacios por observar, se consideró la sugerida en la investigación sobre la creación del conocimiento organizacional realizada por Gorman (2004): (a) directivos, (b) docentes y (c) espacios como reuniones de planificación de los comités de gestión así como rutinas organizativas. A continuación, es necesario detallar la muestra obtenida:

- Para la observación: Reuniones de los comités de gestión, porque son espacios donde los individuos comparten conocimiento tácito que puede convertirse en explícito. Se tomó el 15\% de la población (Cardona, 2002), lo que hace un total de dos comités.

- Para la entrevista individual: Todos los directivos, porque la población solo es de tres y ellos cumplen un rol fundamental tanto 


\section{BETTY ALFARO PALACIOS}

dentro del proceso de creación de conocimiento organizacional como en la identificación del capital estructural. De igual forma, se contó con dos coordinadores de los comités de gestión; se tomó el $15 \%$ de la población (Cardona, 2002). Por otra parte, del total de la población de docentes, se tomó el 10\% (Cardona, 2002), lo que hizo un total de cinco.

- Para el análisis documental: El documento de gestión a largo plazo. El PEI se consideró conocimiento explícito sistematizado de la institución educativa.

En la metodología se han utilizado varias técnicas. Esto permitió hacer la triangulación en el momento del análisis y darle mayor riqueza interpretativa a los resultados. Las técnicas empleadas fueron las propias de este enfoque investigativo: (a) la observación, (b) la entrevista en profundidad y (c) el análisis documental (Denzin \& Lincoln citados en Vieytes, 2004). Además, para poder tener acceso al "campo de estudio", se obtuvo el permiso de las autoridades de la organización educativa y se ofreció la entrega de los resultados de la investigación al término de esta.

Se contó con el apoyo de un "portero" de este grupo (Vieytes, 2004) quien nos puso en contacto con los coordinadores de los comités de gestión y con algunas fechas en las que se iban a realizar reuniones y la jornada de capacitación que se observó, lo que nos permitió una estadía siempre fructífera. Todo esto nos dio la oportunidad de conocer el campo y establecer contactos. La fecha y hora de las entrevistas fueron establecidas entre la investigadora y los entrevistados, y el documento de gestión analizado nos fue entregado sin dificultad. La información fue recogida por la misma investigadora. 


\section{LA GESTIÓN DEL TALENTO PARA PRODUCIR Y GESTIONAR EL CAPITAL INTELECTUAL O CONOCIMIENTO EXPLÍCITO EN NUESTRAS ESCUELAS}

Asimismo, la observación cualitativa realizada, según el grado de participación, fue participante como espectador y, según la apertura, fue abierta (Vieytes, 2004) "estando allí y en medio de la acción", sin formar parte de ella y sin ocultar la tarea. Esto hizo posible recoger la información de primera mano y sin modificarla (Cardona, 2002). La experiencia consistió en participar de la vida cotidiana de la institución —en los inicios del mes de julio hasta comienzos del mes de setiembre del 2006 — para poder conocer lo que allí acontecía con lo que respecta al tema que se estaba investigando.

Se observaron los espacios brindados por las reuniones de planificación de los comités de gestión; una jornada de capacitación brindada por la Central del Movimiento Fe y Alegría con motivo de cumplir 40 años tanto el Movimiento como la institución; una jornada de compartir trabajos elaborados con un software educativo (Clic 3,0) y algunas otras rutinas organizativas como las llevadas a cabo los días sábados por el Comité de Voluntariado. De esta manera, la permanencia en la escuela pudo ser en cualquier horario y en cualquier día de la semana. Estos incluyeron los días sábados para ganar confianza y simpatizar con la gente, y poder recoger información valiosa a fin de darle mayor profundidad al dato recogido (Vieytes, 2004).

Las primeras aproximaciones al campo permitieron tener una idea holística de lo que acontecía en la escuela con respecto al tema de investigación. En consecuencia, se pudo tener observaciones más focalizadas hacia lo que se quería obtener. Asimismo, permitió hacer las descripciones cada vez más puntuales.

La observación se hizo libremente, guiada por los objetivos de estudio (Vieytes, 2004). Además, la información se recogió en notas de registro permanentes y se hicieron tanto durante la observación como inmediatamente después de haberse realizado. Estos registros fueron de tipo 


\section{BETTY ALFARO PALACIOS}

narrativo-descriptivo e intentaron describir la conducta en el contexto, que según Vieytes son propios de la investigación cualitativa exploratoria que se realizó. Se anotaron tanto el tiempo como las circunstancias. En este sentido el tiempo fue variable desde lo mínimo, que llevó 25 minutos, hasta lo máximo que fue de ocho horas, tiempo que duró la jornada de capacitación de docentes. Los registros de las observaciones realizadas se han codificado de manera simple utilizando números correlativos, empezando desde el número uno.

La segunda técnica que se empleó fue la entrevista, propia de la investigación cualitativa, que es flexible y dinámica constituyendo ante todo una narración conversacional (Vieytes, 2004). Las entrevistas se realizaron en el mes de setiembre del 2006 y fueron de tipo individual, pues "constituyen un recurso privilegiado para acceder a la información desde la perspectiva del actor" (Vieytes, 2004, p. 661). Esta técnica nos brindó la posibilidad de esclarecer y seguir las preguntas y respuestas de acuerdo con la información previamente establecida. El formato de la guía de entrevista se asemeja más a una conversación cotidiana que a un intercambio de preguntas y respuestas de investigador(a) y entrevistado(a), guardando siempre la distancia de la conversación cotidiana, dado que se tenía una intención inicial.

De igual modo, los entrevistados prestaron siempre su consentimiento para realizar las entrevistas. Estas se realizaron siempre buscando el lugar adecuado, lejano de las interrupciones propias del contexto de la escuela como el espacio de los recreos. Estos lugares fueron la biblioteca de la escuela, las oficinas de la dirección o subdirecciones, las aulas en los momentos en que los estudiantes se encontraban realizando otras tareas fuera de ellas y siempre se obtuvo el apoyo tanto de la plana jerárquica como de los docentes. La información brindada se grabó en casetes, para ello se pidió antes la autorización a los entrevistados. 


\section{LA GESTIÓN DEL TALENTO PARA PRODUCIR Y GESTIONAR EL CAPITAL INTELECTUAL O CONOCIMIENTO EXPLÍCITO EN NUESTRAS ESCUELAS}

Aunque en un primer momento se planteó la entrevista grupal para los coordinadores y profesores de los tres niveles, esta no se pudo aplicar porque "en esta institución de lunes a viernes las clases no se interrumpen" (Directora) y los días sábados resultaron difíciles de convocar a los docentes, por lo tanto quedó descartada. De igual manera, hubo oportunidades en que se tuvo que regresar varias veces al campo de investigación para realizar algunas de las entrevistas, ya sea porque la o el informante no estaba presente; o porque surgió de improviso alguna reunión en la escuela. Todo esto dio lugar a que para las 10 entrevistas se tome todo un mes.

Cabe añadir ahora algunos detalles del análisis de la información recogida con esta técnica. Como no se encontraron diferencias relevantes entre las respuestas brindadas por los entrevistados, por jerarquía, género y nivel, las entrevistas han sido ordenadas de manera simple otorgándoles un número (código) a cada una de ellas. Se empezó por el número 1 hasta el número 10, que fue el total de entrevistados(as).

El análisis documental fue la tercera técnica que se utilizó, porque "el lenguaje cristaliza de manera privilegiada el universo simbólico en el que se inscribe y cobra significado la vida de los hombres $y(. .$.$) es un producto$ que expresa la realidad subjetiva y social(...) y es plasmada en diferentes formatos y soportes" (Vieytes, 2004, p. 289). Según Hernández (1998), la unidad de análisis que se analizó ha sido el ítem, como una unidad global. En este sentido, se examinó el documento de gestión representativo de la institución en estudio, el Proyecto Educativo Institucional, para conocer la comunicación que brinda en lo que respecta a las categorías en estudio.

La primera etapa de investigación fue el acercamiento al "campo" para recoger la información mediante la observación, la entrevista y el análisis documental. En la segunda etapa, se categorizó y organizó la información en una Matriz de Organización según las categorías, subcategorías e 
indicadores establecidos de acuerdo a la teoría. En la tercera etapa, se realizó un análisis interpretativo de acuerdo con el marco teórico que sustenta esta investigación, el cual, según el enfoque de investigación cualitativa asumido, se ha hecho a lo largo de todo el proceso de la investigación.

Las citas textuales de los informantes que acompañan a este análisis se han tomado teniendo en cuenta tres aspectos. El primero tiene que ver con la fidelidad a lo dicho por los entrevistados, lo que da veracidad a lo que se afirma en el estudio. El segundo, está referido a la relación entre estas y la teoría que apoya a esta investigación. Finalmente, el tercero tiene como finalidad ilustrar o dar mayor énfasis a aquello que estamos expresando en la interpretación.

\section{Análisis de los Resultados}

El análisis interpretativo de la información acopiada servirá para entender cómo la institución en estudio puede ser un gran espacio donde se pueda llevar a cabo la conversión social del conocimiento. Permitirá conocer, además, de qué manera se lleva a cabo el proceso de creación del conocimiento organizacional o capital intelectual en el Institución Educativa Fe y Alegría N. 2. Esto será posible a través de la aplicación del uso de los modos de conversión del conocimiento explicados anteriormente. 


\subsection{Los modos: permitiendo la conversión del conocimiento Tácito - Explícito - Tácito en la Institución Educativa Fe y Alegría N. 2}

A continuación, se mostrarán de forma detallada cada uno de los modos de conversión de conocimiento utilizados en torno a la Institución Educativa Fe y Alegría N. 2. Estos son los que siguen: (a) socialización, (b) exteriorización, (c) combinación y (d) interiorización. De acuerdo con cada modo, se utilizarán, también, fragmentos pertinentes de las entrevistas realizadas.

\subsubsection{Socialización}

En el proceso de socialización (compartir el conocimiento tácito), que se produce actualmente en esta institución educativa, los espacios que aparecen como una gran característica son los formales. Así, los espacios formales que se establecen en esta escuela son brindados por los comités de gestión, las numerosas jornadas pedagógicas, las reuniones formales y las capacitaciones que se llevan a cabo en la institución. Aquello puede verificarse en el siguiente fragmento:

(...) los espacios están dados a nivel de comités y de repente en jornadas de intercambio pedagógico que hacemos (...) fuera de horario de clases... por ejemplo cuando trabajamos (...) en el área de matemática en Matemática para Todos, allí hay charlas de intercambio pedagógico (...). (Entrevista 4)

Aunque algunos afirman que no lo hagan en espacios muy formales, reconocen también que en la escuela no tienen espacios muy libres para compartir (Entrevista 8) lo que se confirma con lo que otros afirman: “(...) a 


\section{BETTY ALFARO PALACIOS}

veces lo que nos falta es reunirnos más (...)" (Entrevista 7). Esto hace evidente que los docentes necesitan espacios informales, como sostienen Nonaka \& Takeuchi (1995), que ayuden a movilizar el conocimiento tácito. Este es fruto de la experiencia y la pericia pedagógica que poseen los docentes hacia otros niveles de la estructura organizativa.

La frecuencia con que se llevan a cabo las reuniones aparece como una característica diferenciada entre niveles. Así, los docentes de primaria son los que frecuentemente se reúnen. Estas expresiones nos demuestran que en la escuela no está establecida la frecuencia de las reuniones en toda la organización:

(...) los de primaria veo que se reúnen todas las semanas (...) no, nosotros en secundaria, no (...) cada uno (...). (Entrevista 6) (...) al menos en primaria (...) semanalmente nos reunimos, generalmente los viernes, los miércoles, a ver cómo está yendo el aprendizaje de los chicos (...). (Entrevista 5)

Por otro lado, dentro de estos espacios la forma de compartir que emplean los docentes, se encuentra la comunicación cara a cara. Gracias a esta, intercambian ideas a través de la discusión, la lluvia de ideas, la conversación y también hacen uso de la internet para intercambiar información: “(...) el profesor de computación entrega el editorial que la Hermana (...) ha enviado desde España, vía correo electrónico (...)” (Registro de observación 3).

Esta última característica - la internet-, propia de la sociedad del conocimiento, permite complementar lo que los autores de la teoría afirman con respecto a la forma de compartir conocimientos. Esto es, la observación, la lluvia de ideas, el diálogo y se agrega internet. Este medio es una característica considerada por el Proyecto Meritum (2002) como el principal vehículo de difusión del conocimiento y que puede ser aprovechada en la escuela para dar 


\section{LA GESTIÓN DEL TALENTO PARA PRODUCIR Y GESTIONAR EL CAPITAL INTELECTUAL O CONOCIMIENTO EXPLÍCITO EN NUESTRAS ESCUELAS}

inicio a una red de comunicación propuesta por los autores de la teoría en el modo combinación.

Esta red se puede establecer entre los integrantes de la comunidad educativa. Para ello se aprovecha que cuenta con los equipos y el software de computación del Plan Huascarán del Ministerio de Educación y de las Aulas Telemáticas de Fe y Alegría. De esta manera, socialización y combinación son dos modos que pueden interactuar para la creación del conocimiento organizacional de esta escuela.

Otra forma de compartir conocimiento tácito puede darse al exponer las innovaciones que realizan los docentes y que solo quedan a nivel de aula. Por ese motivo, afirman lo siguiente: (...) Nosotros los invitamos a socializar (...) no lo hicieron (...) yo no sé (...) yo he visto sus trabajos y ¿por qué no los presentan? (...)” (Entrevista 1). Esta negación impide que se compartan experiencias a través de la observación que es un elemento clave de la socialización. Al respecto expresan que es una idea que no se les había ocurrido, que generalmente no entran a observar a los demás profesores o que "es muy buena trabajando en el aula (...) pueden observarla si quieren (...) pero no necesariamente (...) (Entrevista 3).

Esta última expresión pone en evidencia que la identificación de docentes con pericia no es práctica que se asuma en la institución para que los demás compañeros observen el desempeño profesional de los docentes expertos y transfieran de esta manera el conocimiento tácito a través de la experiencia. La observación la realizan los directivos como parte del sistema de monitoreo de la institución, pero no se propicia la observación entre pares. Esto permitiría que los docentes adquieran conocimiento tácito, a traves de imitación de sus colegas sin usar el lenguaje, tal como lo afirman los autores de la teoría que sustenta este estudio. 


\section{BETTY ALFARO PALACIOS}

\subsubsection{Exteriorización}

La exteriorización (creando conceptos) en la Institución Educativa, generalmente está ausente de las actividades que se llevan a cabo. Incluso docentes y directivos reconocen que les falta sistematizar las experiencias (Entrevista 1), que tienen una "rica experiencia" que es necesario sistematizar (Documento), que hay experiencias "bonitas" que se pueden sistematizar y sostienen además que no les gusta redactar (Entrevista 2). "El problema que teníamos antes y que ahora lo estamos superando es que muchas de estas cosas no las plasmábamos en documentos (...) hacíamos... hacíamos... y punto...” (Entrevista 3).

La intención de sistematizar las experiencias está expresada en su PEI, como una meta que se proponen. Así, "al 2010 se han sistematizado tres experiencias innovadoras en la línea del PEI" (Documento). Desde luego, el no sistematizar las experiencias se contradice con lo que sostiene la teoría: “(...) escribir es un acto de convertir el conocimiento tácito (...)” (Nonaka \& Takeuchi, 1995, p. 64), y es que la exteriorización es un paso fundamental en la creación del conocimiento organizacional.

Sin embargo, hemos podido darnos cuenta, en nuestra observación en la escuela, que en la institución se brindan ciertos espacios para que este proceso se dé. Entre ellos figuran los talleres de capacitación:

En la observación realizada durante un taller de capacitación, llevado a cabo por los 40 años de la institución(...) los docentes a partir del compartir elaboran carteles con propuestas de cómo enfrentarían los problemas presentados y la pizarra se llena de carteles con las ideas de los docentes(...) la monitora hace un proceso de ir sistematizando, pero lo hace ella sola cuando bien puede ir haciéndolo con los docentes para que 


\section{LA GESTIÓN DEL TALENTO PARA PRODUCIR Y GESTIONAR EL CAPITAL INTELECTUAL O CONOCIMIENTO EXPLÍCITO EN NUESTRAS ESCUELAS}

se convierta en la sistematización del taller(...). (Registro de observación 14)

Más aún, estos espacios no se están utilizando para empezar a producir capital intelectual o conocimiento explícito. Este, como se ha mencionado, surge a partir del diálogo y la reflexión colectiva como requisito fundamental de la exteriorización (Nonaka \& Takeuchi, 1995; Wei Choo, 1999). Como puede observarse, la exteriorización es un proceso que, como afirmaron Nonaka y Takeuchi (1995), también ha sido descuidado en esta institución a pesar de que este aspecto es lo esencial del proceso de creación de conocimiento organizacional, ya que si esto no se da, el conocimiento no puede hacerse tangible. Esto nos permite corroborar, lo que los autores afirman, y es que si el conocimiento tácito y el conocimiento explícito no interactúan, no puede surgir una innovación. Después de la innovación de la Propuesta Curricular no se ha producido otra:

(...) hacemos proyectos de innovación, pero son proyectitos así pequeños $(. .$.$) nosotros hemos participado en algunos seminarios$ de innovación pedagógicos y siempre hemos presentado nuestro proyecto curricular, no hemos hecho otra cosa(...) (Entrevista 2)

En cambio, durante los procesos de creación de conocimiento organizacional que se vivió en la escuela para implementar la Propuesta Curricular y en la elaboración del PEI, los docentes afirman que se definían los conceptos para tenerlos claros (Entrevista 7) y que además se formó una comisión permanente que se encargaba de recoger todo, de comparar la información y de redactar un documento (Entrevista 4). Esto es coincidente con lo que sostiene la teoría, pues el conocimiento tácito de los docentes interactuó 


\section{BETTY ALFARO PALACIOS}

con el conocimiento explícito para producir la innovación que se está aplicando actualmente en la escuela.

Por otro lado, docentes y directivos sostienen que en la institución se usan algunas metáforas — palabras o frases - para ayudar, por ejemplo, a terminar los trabajos que se empiezan: "Lo que se empieza se termina" (Entrevista 3). De igual forma, usan estas herramientas para que se comprometan a "hacerlo bien”, como ellos afirman (Entrevista 4). No obstante, estas metáforas no se están utilizando directamente para realizar el proceso de exteriorización y ayudar a que el proceso de sistematización se lleve a cabo, con lo cual se produzca el capital estructural de la escuela.

Las metáforas, como conocimiento tácito que se vuelve explícito a través de un lema, son herramientas importantes para crear la interacción y la reflexión entre los individuos (Nonaka \& Takeuchi, 1995). Cabe hacer, entonces, determinadas preguntas. Estas son las que siguen: (a) ¿qué metáforas podrían llevar a esta institución a elaborar capital intelectual que le permita alcanzar los objetivos estratégicos y la visión que se han planteado en su PEI?, (b) ¿quién o quiénes deberían crear estas metáforas? y (c) ¿con base en qué las deben crear?

\subsubsection{Combinación}

Este proceso de comparación de conocimientos explícitos para producir un nuevo conocimiento, se analizará también, en los dos momentos, el actual y el que se vivió durante la creación del conocimiento organizacional para crear la Propuesta Curricular y el PEI. En este último momento, este modo se evidenció cuando los docentes trabajaban en comisiones en las cuales se “compartía la propuesta”, la escribían (Entrevista 7). Además, se hacían síntesis y comparaciones con lo que habían elaborado anteriormente y con base en

ello se hacían las modificaciones y elaboraban un nuevo informe, permitiendo 


\section{LA GESTIÓN DEL TALENTO PARA PRODUCIR Y GESTIONAR EL CAPITAL INTELECTUAL O CONOCIMIENTO EXPLÍCITO EN NUESTRAS ESCUELAS}

incorporar varios conocimientos explícitos. Sostienen además, que fue un largo proceso (Entrevista 8). Todo esto es coincidente con lo que sostiene la teoría, respecto al modo combinación.

Actualmente, el proceso de combinación (construir un arquetipo) en la institución en estudio queda como planteamiento desde el PEI, y es que este proceso está íntimamente ligado al de exteriorización. Por lo tanto, si en la escuela no se da la exteriorización no se puede dar la combinación. Son muchos los arquetipos que se sugieren desde este documento de gestión:

Plan de tutoría de padres de familia(...) plan de atención a los alumnos con problemas(...) plan de voluntariado(...) plan de pastoral (...) plan de lectura(...) plan del área de robolat(...) plan de monitoreo. (Documento)

La experiencia que se ha sistematizado es la que ha dado origen al actual documento del PEI y que ha surgido del anterior. En lo referente a la Propuesta Curricular sostienen: "Bueno un poco lo tenemos expresado en el Proyecto curricular,(...) todos los pasos que tiene que seguir(...)" (Entrevista 2). Es decir, no han logrado elaborar un documento para esta experiencia e incluso consideran que está redactada de manera "esquemática".

Esto permite ver que no es práctica institucionalizada el crear arquetipos de las innovaciones que se producen en la escuela. En esta institución la sistematización, de arquetipos queda reducida a los documentos que son "obligatorios", más que a escribir o a documentar las "mejores prácticas" o innovaciones de la organización. Esta práctica permitiría distribuir el conocimiento a través de todos los integrantes de la organización, como una actividad clave del proceso de gestión del conocimiento planteada por Vásquez (2005). 


\section{BETTY ALFARO PALACIOS}

El hecho de no elaborar un arquetipo se contradice con lo que afirman los autores de la teoría. Por esta razón, no permite que el conocimiento explícito sea reconfigurado al añadir, combinar y categorizar el conocimiento explícito para elaborar un nuevo conocimiento. Para estimular dicho proceso, esta organización puede hacer uso de las TIC con que cuenta y que promueve desde el PEI: "Incrementar el uso de las TIC por los docentes aprovechando la capacitación de las aulas telemáticas”(Documento).

En este sentido, el uso creativo de la red computarizada —en el que juegan un rol importante los middle managers o subdirectoras en el caso de esta escuela - es una herramienta de gran ayuda para que la institución sea innovadora y pueda hacer llegar el conocimiento a quien lo necesita (Vásquez, 2005). Además, será capaz de crear valor a partir de su capital intelectual (Sullivan, 2001). Por otra parte, la institución educativa brinda espacios, generados por las capacitaciones, que se pueden aprovechar para promover este proceso. En estos espacios no se comparan, añaden o combinan los productos que se obtienen:

La monitora lee las propuestas de cada grupo, pero no hace una confrontación con todos los docentes para sacar un mejor producto en cada caso. (Registro de observación 9)

Así, también en otros momentos dentro de los comités de gestión esto no es práctica que se aplique por igual. De esto se desprende la siguiente afirmación: "(...)Le informa que hay una revista en la cual se pueden basar pero no está al alcance de ellas(...)" (Registro de observación 1). A diferencia de lo que se observó en otro comité, para elaborar un nuevo producto, por ejemplo la guía de monitoreo, utilizaron como base la que se encuentra en el PEI. 


\section{LA GESTIÓN DEL TALENTO PARA PRODUCIR Y GESTIONAR EL CAPITAL INTELECTUAL O CONOCIMIENTO EXPLÍCITO EN NUESTRAS ESCUELAS}

\subsubsection{Interiorización}

La interiorización o conversión del conocimiento explícito a tácito, la analizaremos desde los dos momentos: el histórico y el actual. Así, en el momento en que se llevaba a cabo la creación de la propuesta, el proceso de interiorización se realizó al mismo tiempo en que los docentes estaban llevando a cabo el aprendizaje de esta. De esa manera, cumplieron con el principio de "aprender haciendo" que se expresa en la teoría:

(...) ya nosotros lo empezamos a utilizar(...)o sea a la vez que íbamos estudiando ya(...) nosotros ese año lo empezamos a trabajar, pero a la medida que íbamos(...)conociendo, íbamos también trabajando con los chicos(...). (Entrevista 9)

(...)íbamos y preguntábamos, luego nosotras programábamos y ellas las revisaban para ver si estaban de acuerdo(...)los talleres los llevábamos en marzo y ya después teníamos que aplicar(...). (Entrevista 10) 


\section{BETTY ALFARO PALACIOS}

(...)nosotros creemos de que toda la conceptualización que tenemos del aprendizaje aterriza en el aula, entonces el aula es el centro donde se puede constatar si está trabajando así o no. (Entrevista 3)

(...) que tiene que lograrse con el trabajo de los maestros en el aula(...) si bien es cierto hay un proyecto educativo(...)también es cierto que ese proyecto educativo no es para que esté escrito allí sino que se logra(...)se va logrando a través de los maestros en el aula. (Entrevista 8)

Un punto importante que hemos encontrado es que, tanto directivos como docentes, expresan que por más capacitación que reciban esta no es llevada a la práctica, pues "una cosa es el papel” y otra cuando van a clase (Entrevista 6). Para ellos, todavía falta adaptarla en el aula y, más aún, debe haber un compromiso de los docentes para aplicarla, adaptarla y lograrla en el aula (Entrevista 7). En este sentido, si no hay aplicación no se está permitiendo que el conocimiento explícito se vuelva tácito. En último término, en la institución no existen manuales o documentos que ayuden a transferir el conocimiento explícito a otras personas, con lo que se pueda facilitar este proceso.

\section{Conclusión}

Actualmente, el Ministerio de Educación está promoviendo en los directores de las Instituciones Educativas (I.E.) la gestión pedagógica. Esta investigación bien puede servir a las I.E. para promover la gestión del talento de sus docentes, la creación y el compartir del conocimiento tácito o capital intelectual. Esto, por su parte, será posible a través de la gestión del talento de los profesores de la organización educativa. 


\section{LA GESTIÓN DEL TALENTO PARA PRODUCIR Y GESTIONAR EL CAPITAL INTELECTUAL O CONOCIMIENTO EXPLÍCITO EN NUESTRAS ESCUELAS}

\section{Referencias}

Alles, M. (2005). Desarrollo del talento humano basado en competencias. Buenos Aires, Argentina: Granica.

Cañibano, L., Sánchez, P., García-Ayuso, M. \& Chaminade, C. (Eds.). (2002). Guidelines for Managing and Reporting on Intangibles (Intellectual Capital Report). Proyecto Meritum, Universidad Autónoma de Madrid. Recuperado de http://www.uam.es/ proyectosinv/meritum/Link\%20Guidelines\%20Meritum/ version $\% 20$ inglesa $\% 20$ completa.pdf

Capella, J. (2005). Gestión del conocimiento en el campo educativo. Material de Trabajo (3. ${ }^{a}$ ed. rev.). Lima, Perú: Escuela de Graduados, Maestría en gestión de la educación, Pontificia Universidad Católica del Perú. Cardona, M. (2002). Introducción a los métodos de investigación en educación.

Madrid, España: Editorial EOS.

Consejo Nacional de Educación. (2005). Hacia un Proyecto Educativo Nacional. Lima, Perú: Tarea, Asociación Gráfica Educativa.

Consejo Nacional de Educación. (2006). Proyecto Educativo Nacional. Recuperado de http://www.cne.gob.pe/docs/cne-pen/PEN-Oficial.pdf 


\section{BETTY ALFARO PALACIOS}

Edvinsson, L. \& Malone, M. (1999). El capital intelectual. Cómo identificar y calcular el valor de los recursos intangibles de su empresa. Barcelona, España: Ediciones Gestión 2000.

Gimeno Sacristán, J. (2001). Educar y convivir en la cultura global. Madrid, España: Ediciones Morata.

Grant, R. (1997). The Knowledge-Based View of the Firm: Implications for Management Practice. Long Range Planning, (3), 450-454.

Hargreaves, A. (2003). Enseñar en la sociedad del conocimiento. Barcelona, España: Ediciones Octaedro.

Hernández, R. \& Fernández, C. (1998). Metodología de la Investigación. México D.F., México: McGraw-Hill Interamericana Editores.

Jericó, P. (2001). Gestión del talento. Del profesional con talento al talento organizativo. Madrid, España: Financial Times - Prentice Hall.

Kelleher, D. (2000). Knowlwdge Management-An Evolving Professional Competence. En Fesabid. (Ed.) La gestión del conocimiento: retos y soluciones de los profesionales de la información (pp. 19-20). Bilbao, España: Servicio Editorial de la Universidad del País Vasco.

Ley 28044. Ley General de Educación. Congreso de la República (2003). Ministerio de Educación del Perú. (2005). Diseño Curricular Nacional de Educación Básica Regular. Proceso de Articulación. Lima, Perú: DINEIP- DINESST. 


\section{LA GESTIÓN DEL TALENTO PARA PRODUCIR Y GESTIONAR EL CAPITAL INTELECTUAL O CONOCIMIENTO EXPLÍCITO EN NUESTRAS ESCUELAS}

Nonaka, I. \& Takeuchi, H. (1995). The Knowledge-Creating Company. New York, Oxford, USA: Oxford University Press.

Pricewaterhousecoopers. (2000). La gestión del conocimiento: El tercer factor.

Recuperado de http://www.pweglobal.com/es/esp/ins-so/spec-int/ El_tercer_factor.PDF

Proyecto Regional de la Educación para América Latina y el Caribe. (2004).

PRELAC. Un trayecto regional hacia la Educación para Todos. Revista PRELAC, (0), 7-10.

Solé, F. \& Olea de Cárdenas, M. (2002). La formación, la gestión del conocimiento y los intangibles en las organizaciones. En Gestión del conocimiento. Pautas y lineamientos generales (pp. 25-46). Salamanca, España: Dirección General de la Asociación Universitaria Iberoamericana de Postgrado.

Sullivan, P. (2001). Rentabilizar el capital intelectual. Técnicas para optimizar el valor de la innovación. Barcelona, España: Ediciones Paidós Ibérica.

Sveiby, K. (2000). Capital Intelectual. La nueva riqueza de las empresas. (García, A. Trad.) Paris, Francia: Maxima Laurent du Mesnil Ëditeur.

TFPL Ltd. (1999). Skills for knowledge management: Building a knowledge economy: A report by TFPL Ltd Based on research jointly funded by TFPL and the Library and Information Commission. London, United Kingdom: TFPL Ltd. 


\section{BETTY ALFARO PALACIOS}

Vásquez, B. S. (2005). La Gestión del conocimiento. En Villafane, J. (Ed.) La comunicación empresarial y la gestión de los intangibles en España y Latinoamérica (pp. 31 - 45). Madrid, España: Pirámide.

Vicario, S. C. (2002). Gestión de conocimiento, desafío de la educación. Recuperado de http://www.somece.org.mx/memorias/2002/ Grupo5/Vicario.doc

Vieujean, J. (1959). Humanismo y Educación. Pedagogía del conocimiento integral. Buenos Aires, Argentina: Ediciones Humanismo.

Vieytes, R. (2004). Metodología de la investigación en organizaciones, mercado $y$ sociedad. Buenos Aires, Argentina: Editorial de las ciencias.

Wei Choo, Ch. (1999). La organización inteligente: el empleo de la información para dar significado, crear conocimiento y tomar decisiones. (Rey, D. Trad.). México D.F., México: Oxford University Press.

Wiig, K. (1997). Integrating Intellectual Capital and Knowledge Management. En Long Range Planning, (3), 399-405. 